\title{
Hamiltonian description of self-consistent wave-particle dynamics in a periodic structure
}

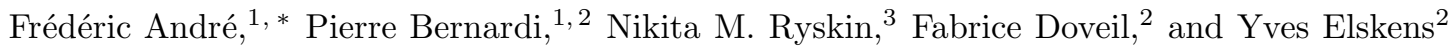 \\ ${ }^{1}$ Thales Electron Devices, rue Latécoère, 2, FR-78140 Vélizy, France \\ ${ }^{2} U M R 7345$ CNRS-Aix-Marseille-Université, campus Saint-Jérôme, case 321, \\ av. esc. Normandie-Niemen, FR-13397 Marseille cedex 20 \\ ${ }^{3}$ Saratov State University, Saratov 410012, Russia
}

(Dated: September 6, 2018)

\begin{abstract}
Conservation of energy and momentum in the classical theory of radiating electrons has been a challenging problem since its inception. We propose a formulation of classical electrodynamics in Hamiltonian form that satisfies the Maxwell equations and the Lorentz force. The radiated field is represented with eigenfunctions using the Gel'fand $\beta$-transform. The electron Hamiltonian is the standard one coupling the particles with the propagating fields. The dynamics conserves energy and excludes self-acceleration. A complete Hamiltonian formulation results from adding electrostatic action-at-a-distance coupling between electrons.

PACS numbers: 84.40.Fe (microwave tubes)

52.35.Fp (Plasma: electrostatic waves and oscillations)

52.40. $\mathrm{Mj}$ (particle beam interaction in plasmas)

52.20.Dq (particle orbits)

Keywords : wave-particle interaction, traveling wave tube, $\beta$-transform, Floquet boundary condition
\end{abstract}

To model consistently the interaction between electrons and waves in devices such as traveling wave tubes, free electron lasers or synchrotrons, we are presently left with two options. The first [6, 11, 17] is to consider the flow of electrons as a distributed charge and current density coupled with the field through the Maxwell equations. Since it generates diverging singularities, the particle nature of electrons is intentionally overlooked until the question of determining the trajectories of the flow is raised. For the latter, the only possibility is to return to a particle description in which the Lorentz force applies. This change of model for the flow precludes the description of the wave-electron system in Hamiltonian form. One reason is that a procedure is needed to distribute the electron charge and current into a finite volume. This procedure, usually based on meshing space, can only be arbitrary. The second option [8, 10, 13] is to consistently consider electrons as particles and to determine the field they radiate starting from the Liénard-Wiechert potentials. Dirac's [4] sharp analysis indeed provides an accurate determination of the reaction from the radiated field in the limit of a point electron, yet this approach leads to serious difficulties [4, 10, 13, 16, 18]; among these an infinite rest mass of the electron, self-acceleration and acausality, also appear incompatible with the existence of a well-posed Hamiltonian.

Hamiltonians are essential to consistently define energy and momentum. They also have great practical usefulness to find approximate solutions in complex systems or to control errors in numerical integration schemes [7]. So their absence in the case of the classical wave-electron interaction is both theoretically and practically unsatisfactory. While the final solution to these problems may involve (upgraded, regular) quantum electrodynamics, one could at least hope for a consistent classical approximation, which would be the classical limit of its quantum counterpart. For instance, in the limit where the electron radiates a large number of photons, each of them having a small energy compared with its kinetic energy, which is the case for the aforementioned devices, one could expect that averaging the quantum theory over a large number of emitted photons would provide a consistent classical limit. But such a theory, free of the above problems, remains to be found [9, 16, 20, 21].

To progress in this direction, we undertake the construction of a Hamiltonian in situations where the boundary conditions are periodic in one or more dimensions, and we focus on the radiated field modes interacting with the electrons. Our method applies both to traveling wave tubes, meant to amplify "slow" modes in the periodic structure, and to free electron lasers where the electrons couple to "fast" modes.

First of all, one can distinguish the space-charge field, which is the curl-free part of the fields, from the radiated field, which is the divergence-free part. In free space, the space-charge field between several electrons with positions $\mathbf{r}_{i}$ derives from the Hamiltonian $\mathcal{H}_{\mathrm{sc}}=$ $\frac{1}{8 \pi \epsilon_{0}} \sum_{i \neq j} e^{2} /\left\|\mathbf{r}_{i}-\mathbf{r}_{j}\right\|$. Adopting this point of view eliminates the problem of infinite rest mass, by eliminating altogether the electrostatic field degrees of freedom. So one is left with finding a Hamiltonian for the radiated fields coupled with the electrons. This is the object of this work.

The geometry is periodic along the $x$ axis with period $d$ (extension to two- and three-dimensional lattices is straightforward). The lattice may include perfect metal $\left(\mathbf{E} \times \mathbf{e}_{\perp}=\mathbf{0}, \mathbf{H} \cdot \mathbf{e}_{\perp}=0\right)$ boundary condition. All equations are written in the reference frame of these bound- 
ary conditions. All periods, with shape $\mathcal{V}_{0}$ and volume $\left|\mathcal{V}_{0}\right|$, form a domain $\mathcal{V}_{\mathbb{Z}}:=\cup_{n \in \mathbb{Z}}\left\{\mathbf{r}+n d \mathbf{e}_{x}: \mathbf{r} \in \mathcal{V}_{0}\right\}$. The propagating waves are described with their electric and magnetic fields, $\mathbf{E}(\mathbf{r}, t), \mathbf{H}(\mathbf{r}, t)$, obeying the Maxwell equations with sources [2].

We decompose the radiated field on the function basis made of the propagating modes. These modes satisfy the metal boundary condition on cavity walls, and the Floquet condition, $\mathbf{E}\left(\mathbf{r}+d \mathbf{e}_{x}\right)=\mathrm{e}^{-\mathrm{i} \beta d} \mathbf{E}(\mathbf{r})$ for some $\beta \in$ $\mathbb{R}$. Inside $\mathcal{V}_{0}$, they satisfy $\nabla \cdot \mathbf{E}_{s \beta}=0, \nabla \cdot \mathbf{H}_{s \beta}=0$, and

$$
\begin{aligned}
\operatorname{rot} \mathbf{E}_{s \beta} & =-\mathrm{i} \mu_{0} \Omega_{s \beta} \mathbf{H}_{s \beta} \\
\operatorname{rot} \mathbf{H}_{s \beta} & =\mathrm{i} \epsilon_{0} \Omega_{s \beta} \mathbf{E}_{s \beta}
\end{aligned}
$$

where the $\Omega_{s \beta}$ are the real eigenvalues, $\mu_{0}$ and $\epsilon_{0}$ the permeability and permittivity of vacuum. For a given $\beta \in\left[0,2 \pi / d\left[\right.\right.$, the set of eigen electric fields $\mathbf{E}_{s \beta}(s \in \mathbb{N})$ is an orthogonal basis of the divergence-free subset of $H\left(\operatorname{rot}, \mathcal{V}_{0}\right)$ (this is the Hilbert space of square-integrable fields on $\mathcal{V}_{0}$ which have a square-integrable curl). Any divergence-free field $\mathbf{E}_{\beta}(\mathbf{r}, t)$ on $\mathcal{V}_{0}$ can be decomposed on the $\mathbf{E}_{s \beta}$ with coefficients $V_{s \beta}$,

$$
\mathbf{E}_{\beta}(\mathbf{r}, t)=\sum_{s \in \mathbb{N}} V_{s \beta}(t) \mathbf{E}_{s \beta}(\mathbf{r}) .
$$

This decomposition can be continued to $\mathcal{V}_{\mathbb{Z}}$ thanks to the Floquet condition, and the continuation field satisfies the Floquet condition as well (possibly with a Gibbs phenomenon, depending on the field regularity).

An arbitrary function $G$ in the propagating structure does not, in general, satisfy the Floquet condition. But it can be expanded on functions $G_{\beta}$ satisfying the Floquet condition. Indeed, the Fourier series on the variable $\beta$, with $x$ as a parameter,

$$
G_{\beta}(x, t):=\sum_{n \in \mathbb{Z}} G(x+n d, t) \mathrm{e}^{\mathrm{i} n \beta d}
$$

satisfies the Floquet condition with respect to $x$. The $n$-th Fourier coefficient in the series is given by

$$
G(x+n d, t)=\frac{1}{2 \pi} \int_{\beta d=0}^{2 \pi} G_{\beta}(x, t) \mathrm{e}^{-\mathrm{i} n \beta d} \mathrm{~d}(\beta d),
$$

which for $n=0$ yields exactly the requested expansion. The transform (4), hereafter referred to as the $\beta$ transform [5, 12, 15, 19], decomposes any function into a superposition of functions satisfying the Floquet condition. It will be central in what follows. Relation (5) is the inverse $\beta$-transform.

Using eq. (3), we finally obtain

$$
\mathbf{E}(\mathbf{r}, t)=\frac{1}{2 \pi} \sum_{s \in \mathbb{N}} \int_{\beta d=0}^{2 \pi} V_{s \beta}(t) \mathbf{E}_{s \beta}(\mathbf{r}) \mathrm{d}(\beta d) .
$$

This decomposition holds for any free electric field in the structure, e.g. propagating modes, evanescent modes or superposition of these. The magnetic field expansion is written

$$
\mathbf{H}_{\beta}(\mathbf{r}, t)=\mathrm{i} \sum_{s \in \mathbb{N}} I_{s \beta}(t) \mathbf{H}_{s \beta}(\mathbf{r})
$$

with the factor i for later convenience.

Our initial choice to consider the propagating field independently holds only if it is decoupled from the spacecharge field. This latter adds the curl-free term $-\nabla \phi$ to the expression of $\mathbf{E}$ where $\phi$ satisfies the Poisson equation $\Delta \phi=-\rho / \epsilon_{0}$. The $\beta$-transform commutes both with time and space derivatives, so Maxwell equations are valid for $\mathbf{E}_{\beta}$ and $\mathbf{H}_{\beta}$ as well. Using the $\beta$-transform of $\phi$, the general form of the electric field in the presence of charged particles is

$$
\mathbf{E}_{\beta}(\mathbf{r}, t)=\sum_{s \in \mathbb{N}} V_{s \beta}(t) \mathbf{E}_{s \beta}(\mathbf{r})-\nabla \phi_{\beta} .
$$

Using Maxwell equations, the field decompositions (8) and (77), and the definition of eigenfields $\mathbf{E}_{s \beta}$ and $\mathbf{H}_{s \beta}$, we obtain

$$
\begin{aligned}
-\sum_{s} I_{s \beta} \Omega_{s \beta} \mathbf{E}_{s \beta} & =\sum_{s} \dot{V}_{s \beta} \mathbf{E}_{s \beta}+\frac{\mathbf{J}_{\beta}}{\epsilon_{0}}-\frac{\partial \nabla \phi_{\beta}}{\partial t}, \\
\sum_{s} V_{s \beta} \Omega_{s \beta} \mathbf{H}_{s \beta} & =\sum_{s} \dot{I}_{s \beta} \mathbf{H}_{s \beta},
\end{aligned}
$$

where $\mathbf{J}$ is the current density. We multiply the first expression by the conjugate of the field $\mathbf{E}_{s^{\prime} \beta}^{*}$ and the second expression by $\mathbf{H}_{s^{\prime} \beta}^{*}$. Integrating the resulting expressions over the volume $\mathcal{V}_{0}$ yields vanishing terms for $s \neq s^{\prime}$. Otherwise we exhibit the electric $\frac{1}{2} \epsilon_{0} \int_{\mathcal{V}_{0}}\left|\mathbf{E}_{s \beta}\right|^{2} \mathrm{~d}^{3} \mathbf{r}$ and magnetic $\frac{1}{2} \mu_{0} \int_{\mathcal{V}_{0}}\left|\mathbf{H}_{s \beta}\right|^{2} \mathrm{~d}^{3} \mathbf{r}$ energies stored in one period for the corresponding propagation mode. Both energies are equal to half the total stored energy $N_{s \beta}$ of the basis function. The volume integral involving $\nabla \phi_{\beta}$ is transformed to a surface integral thanks to the identity $\nabla \cdot\left(\phi_{\beta} \mathbf{E}_{s^{\prime} \beta}^{*}\right)=\left(\nabla \phi_{\beta}\right) \cdot \mathbf{E}_{s^{\prime} \beta}^{*}+\phi_{\beta} \nabla \cdot \mathbf{E}_{s^{\prime} \beta}^{*}$. The electric field basis functions are divergence-free, so only the surface integral $\int_{\partial \mathcal{V}_{0}} \phi_{\beta} \mathbf{E}_{s^{\prime} \beta}^{*} \cdot \mathbf{e}_{\perp} \mathrm{d} S$ remains. The surface integral over the two cross-sections of the waveguide vanishes because both $\phi_{\beta}$ and $\mathbf{E}_{s^{\prime} \beta}$ satisfy the Floquet condition. The lateral metallic parts are at imposed time-invariant potentials, a property $\phi_{\beta}$ inherits, so the time derivative of the corresponding surface integral vanishes. This concludes our demonstration that the evolution of the $V_{s \beta}$ and $I_{s \beta}$ representing the propagating field is decoupled from the space-charge field

$$
\begin{aligned}
& \dot{V}_{s \beta}+\Omega_{s \beta} I_{s \beta}=-\frac{1}{N_{s \beta}} \int_{\mathcal{V}_{0}} \mathbf{J}_{\beta} \cdot \mathbf{E}_{s \beta}^{*} \mathrm{~d}^{3} \mathbf{r}, \\
& \dot{I}_{s \beta}-\Omega_{s \beta} V_{s \beta}=0 .
\end{aligned}
$$

We perform the inverse $\beta$-transform of (11) and (12). Like the usual Fourier coefficients, the $\beta$-transform of a 
product is the convolution of its factor transforms. The source term in Maxwell-Ampère is transformed by (4) into an integral over the complete volume $\mathcal{V}_{\mathbb{Z}}$ :

$$
\begin{array}{r}
\int_{\mathcal{V}_{0}} \mathbf{J}_{\beta} \cdot \mathbf{E}_{s \beta}^{*} \mathrm{~d}^{3} \mathbf{r}=\int_{\mathcal{V}_{0}} \sum_{n} \mathbf{J}\left(\mathbf{r}+n d \mathbf{e}_{x}\right) \cdot \mathbf{E}_{s \beta}^{*}(\mathbf{r}) \mathrm{e}^{\mathrm{i} n \beta d} \mathrm{~d}^{3} \mathbf{r} \\
=\int_{\mathcal{V}_{0}} \sum_{n} \mathbf{J}\left(\mathbf{r}+n d \mathbf{e}_{x}\right) \cdot \mathbf{E}_{s \beta}^{*}\left(\mathbf{r}+n d \mathbf{e}_{x}\right) \mathrm{d}^{3} \mathbf{r} \\
=\int_{\mathcal{V}_{\mathbb{Z}}} \mathbf{J} \cdot \mathbf{E}_{s \beta}^{*} \mathrm{~d}^{3} \mathbf{r},
\end{array}
$$

where the second equality follows from the Floquet property of $\mathbf{E}_{s \beta}$. Finally, using the inverse $\beta$-transform,

$$
\begin{aligned}
& \dot{V}_{s n}+\sum_{m} \Omega_{s m} I_{s, n-m}=-\int_{\mathcal{V}_{\mathbb{Z}}} \mathbf{J} \cdot \mathbf{F}_{s,-n} \mathrm{~d}^{3} \mathbf{r}, \\
& \dot{I}_{s n}-\sum_{m} \Omega_{s m} V_{s, n-m}=0
\end{aligned}
$$

where

$$
\mathbf{F}_{s,-n}:=\frac{1}{2 \pi} \int_{0}^{2 \pi} \frac{\mathbf{E}_{s \beta}^{*}}{N_{s \beta}} \mathrm{e}^{\mathrm{i} n \beta d} \mathrm{~d}(\beta d)
$$

whose $\beta$-transform is $\mathbf{F}_{s \beta}=\mathbf{E}_{s \beta} / N_{s \beta}$.

The electric and magnetic fields, given by

$$
\begin{aligned}
\mathbf{E}(\mathbf{r}, t) & =\sum_{s, n} V_{s n}(t) \mathbf{E}_{s,-n}(\mathbf{r})-\nabla \phi(\mathbf{r}, t), \\
\mathbf{H}(\mathbf{r}, t) & =\mathrm{i} \sum_{s, n} I_{s n}(t) \mathbf{H}_{s,-n}(\mathbf{r}),
\end{aligned}
$$

are rewritten

$$
\begin{aligned}
\mathbf{E}(\mathbf{r}, t) & =\sum_{s, n} V_{s n}(t) \mathbf{E}_{s 0}\left(\mathbf{r}-n d \mathbf{e}_{x}\right)-\nabla \phi(\mathbf{r}, t), \\
\mathbf{H}(\mathbf{r}, t) & =\mathrm{i} \sum_{s, n} I_{s n}(t) \mathbf{H}_{s 0}\left(\mathbf{r}-n d \mathbf{e}_{x}\right)
\end{aligned}
$$

Each eigenfunction $\mathbf{H}_{s \beta}$ derives from a vector potential $\mathbf{A}_{s \beta}$

$$
\mu_{0} \mathbf{H}_{s \beta}=\operatorname{rot} \mathbf{A}_{s \beta} .
$$

The inverse $\beta$-transform yields $\mu_{0} \mathbf{H}_{s n}=\operatorname{rot} \mathbf{A}_{s n}$. Using (7), we find

$$
\mathbf{A}=\mathrm{i} \sum_{s, n} I_{s n} \mathbf{A}_{s,-n}+\nabla u
$$

where $u$ is any function of space corresponding to a gauge choice.

These expressions offer an interpretation for the index $n$. The electric field at a particular position is the superposition of the field shape $\mathbf{E}_{s 0}$ generated by the adjacent cells of the periodic structure with amplitudes $V_{s n}$. Therefore $V_{s n}\left(\right.$ resp. $\left.I_{s n}\right)$ can be seen as the contribution of the $s$-th eigenmode in cell $n$ to the electric (resp. magnetic) field. The propagation is described by linearly coupled harmonic oscillators. Cell $n$ is coupled with cell $m$ through the coefficient $\Omega_{s, n-m}=\frac{1}{2 \pi} \int_{-\pi}^{\pi} \Omega_{s \beta} \mathrm{e}^{\mathrm{i}(n-m) \beta d} \mathrm{~d}(\beta d)$, viz. the corresponding Fourier coefficient of the dispersion curve $\Omega_{s \beta}$. The reciprocity condition $\Omega_{s,-\beta}=\Omega_{s \beta}$ ensures $\Omega_{s,-n}=$ $\Omega_{s n} \in \mathbb{R}^{+}$. The same condition on the fields reads $\mathbf{E}_{s,-\beta}=\mathbf{E}_{s \beta}^{*}$, therefore $\mathbf{E}_{s n}=\frac{1}{2 \pi} \int_{-\pi}^{+\pi} \mathbf{E}_{s \beta}^{*} \mathrm{e}^{\mathrm{i} n \beta d} \mathrm{~d}(\beta d)=$ $\frac{1}{\pi} \Re\left(\int_{0}^{+\pi} \mathbf{E}_{s \beta}^{*} \mathrm{e}^{\mathrm{i} n \beta d} \mathrm{~d}(\beta d)\right)$ is a vector with real components only. Symmetrically, $\mathrm{iH}_{s n}$ has only real components. In summary, $V_{s n}, I_{s n}, \mathbf{E}_{s n}, \mathbf{F}_{s n}, \mathrm{i} \mathbf{H}_{s n}, \mathrm{i} \mathbf{A}_{s n}, \Omega_{s n}$, $N_{s n}$ are all real quantities.

Starting from this model, we can now construct a Hamiltonian for the electromagnetic field. The standard [8] Hamiltonian $\frac{1}{2} \int_{\mathcal{V}}\left(\epsilon_{0}|\mathbf{E}|^{2}+\mu_{0}|\mathbf{H}|^{2}\right) \mathrm{d}^{3} \mathbf{r}$ results from the Lorentz force and the Maxwell-Ampère equation. In this work, we want to make no assumption on the final form of the electromagnetic Hamiltonian because corrective terms might be needed. Consequently, we cannot use it as a starting point. Instead, the previous development provides a Hamiltonian for the fields alone, independently from how they are coupled with the electrons. The vectors $V_{s}=\left(\ldots V_{s n} \ldots\right)$ and $I_{s}=\left(\ldots I_{s n} \ldots\right)$ represent the state of the fields, and their time evolutions derive from the Hamiltonian (with conjugate variables $V, I)$

$$
\mathcal{H}_{\mathrm{em}}\left(V_{s}, I_{s}\right)_{s \in \mathbb{N}}=\frac{1}{2} \sum_{s}\left(V_{s} Q_{s} V_{s}^{t}+I_{s} Q_{s} I_{s}^{t}\right),
$$

where ${ }^{t}$ denotes vector transpose, and $Q_{s}$ is the infinite matrix with entries $\left(Q_{s}\right)_{n, m}=\Omega_{s, n-m}$ for $(n, m) \in \mathbb{Z}^{2}$. Till now, the basis functions $\mathbf{E}_{s \beta}$ and $\mathbf{H}_{s \beta}$ can have arbitrary physical dimensions. Adopting (22) as a Hamiltonian, however, results in $V_{s} Q_{s} V_{s}^{t}$ and $I_{s} Q_{s} I_{s}^{t}$ being energies. Hence $V_{s}$ and $I_{s}$ have the same dimensions. Eqs. (13)-(14) impose $\Omega_{s n}$ to be a frequency. Therefore $V_{s}$ and $I_{s}$ have the dimension of the square root of an action (energy divided by frequency). The electric and magnetic field have their standard dimensions and $N_{s \beta}$ has the dimension of a frequency.

The Hamiltonian of the electron in the field of a given 4 -potential $(\phi, \mathbf{A})$ is 13 ]

$$
\mathcal{H}_{\mathrm{el}}(\mathbf{p}, \mathbf{r})=\sqrt{m^{2} c^{4}+c^{2}|\mathbf{p}-e \mathbf{A}(\mathbf{r})|^{2}}+e \phi(\mathbf{r})
$$

where $e$ and $m$ are the electron charge and mass, $c$ is the celerity of light, and the momentum $\mathbf{p}$ is the canonical conjugate of the electron position $\mathbf{r}$. The complete Hamiltonian for the fields and $M$ electrons is necessarily the sum of the purely electromagnetic Hamiltonian $\mathcal{H}_{\mathrm{em}}$ with the Lorentz force dynamic Hamiltonian $\mathcal{H}_{\mathrm{el}}$, where A is a function of the $I_{s n}$ as given by (21) and $\phi$ generates 
the space-charge field,

$$
\mathcal{H}\left(\mathbf{p}_{j}, \mathbf{r}_{j}, V_{s}, I_{s}\right)_{\{1 \leq j \leq M, s \in \mathbb{N}\}}=\sum_{j} \mathcal{H}_{\mathrm{el}}\left(\mathbf{r}_{j}, \mathbf{p}_{j}, I_{s}\right)+\mathcal{H}_{\mathrm{em}} .
$$

The complete physics of the system is governed by it, including the source term in the Maxwell-Ampère equation. This source term has not been used to build the Hamiltonian and is equivalent to eq. (13). In the presence of a single electron with position $\mathbf{r}(t)$ and velocity $\mathbf{v}(t)$, we have [13, §29] $\mathbf{J}(\mathbf{r}, t)=e \mathbf{v}(t) \delta(\mathbf{r}-\mathbf{r}(t))$, and (13) simplifies into

$$
\dot{V}_{s n}=-e \mathbf{v} \cdot \mathbf{F}_{s,-n}(\mathbf{r})-\sum_{m} \Omega_{s m} I_{s, n-m} .
$$

Its derivation from $\mathcal{H}$ is obtained considering that $V_{s n}$ is the generalized momentum canonically conjugate to the generalized coordinate $I_{s n}$,

$$
\begin{aligned}
\dot{V}_{s n} & =-\frac{\partial \mathcal{H}}{\partial I_{s n}} \\
& =-\frac{\partial \mathcal{H}_{\mathrm{em}}}{\partial I_{s n}}+\frac{\mathbf{p}-e \mathbf{A}}{\sqrt{m^{2} c^{4}+c^{2}|\mathbf{p}-e \mathbf{A}|^{2}}} \cdot e \mathrm{i} \mathbf{A}_{s,-n} \\
& =e \mathbf{v} \cdot \mathrm{i} \mathbf{A}_{s,-n}(\mathbf{r})-\sum_{m} \Omega_{s m} I_{s, n-m}
\end{aligned}
$$

Here we used the relation $\mathbf{v}=\dot{\mathbf{r}}=\partial \mathcal{H}_{\mathrm{el}} / \partial \mathbf{p}=c^{2}(\mathbf{p}-$ $e \mathbf{A}) / \sqrt{m^{2} c^{4}+c^{2}|\mathbf{p}-e \mathbf{A}|^{2}}$, that derives directly from the electron Hamiltonian (23).

Eqs. (25) and (26) are incompatible unless

$$
\mathbf{F}_{s n}(\mathbf{r})=-\mathrm{i} \mathbf{A}_{s n}(\mathbf{r}) .
$$

The $\beta$-transform gives $\mathbf{E}_{s \beta} / N_{s \beta}=\mathbf{F}_{s \beta}=-\mathrm{i} \mathbf{A}_{s \beta}$. By definition, $\mathbf{E}_{s \beta}=-\mathrm{i} \Omega_{s \beta} \mathbf{A}_{s \beta}-\nabla u_{s \beta}$, so the two equations reconcile when

$$
N_{s \beta}=\Omega_{s \beta} \quad \text { and } \quad \nabla u_{s \beta}=0 .
$$

It is always possible to choose basis functions satisfying the Coulomb gauge, which is the second condition. The first condition clarifies the question of the electromagnetic energy. In this work, it is given by the Hamiltonian $\mathcal{H}_{\mathrm{em}}$ while the usual Poynting energy is $\frac{1}{2} \int_{\mathcal{V}_{\mathbb{Z}}}\left(\epsilon_{0}|\mathbf{E}|^{2}+\mu_{0}|\mathbf{H}|^{2}\right) \mathrm{d}^{3} \mathbf{r}$. In the latter expression, consider the electric part $\mathcal{E}=\frac{1}{2} \epsilon_{0} \int_{\mathcal{V}_{\mathbb{Z}}}|\mathbf{E}|^{2} \mathrm{~d}^{3} \mathbf{r}=$ $\frac{1}{2} \epsilon_{0} \sum_{n} \int_{\mathcal{V}_{0}}\left|\mathbf{E}\left(\mathbf{r}+n d \mathbf{e}_{x}\right)\right|^{2} \mathrm{~d}^{3} \mathbf{r}$. Using Parseval's relation, this is also $\frac{1}{2} \epsilon_{0} \int_{\mathcal{V}_{0}} \int_{0}^{2 \pi}\left|\mathbf{E}_{\beta}\right|^{2} \mathrm{~d}(\beta d) \mathrm{d}^{3} \mathbf{r}$. Now, using the decomposition (8) of the electric field, we find $\mathcal{E}=\frac{1}{2} \epsilon_{0} \int_{\mathcal{V}_{0}} \int_{0}^{2 \pi}\left|\sum_{s} V_{s \beta} \mathbf{E}_{s \beta}\right|^{2} \mathrm{~d}(\beta d) \mathrm{d}^{3} \mathbf{r}$. Orthogonality of the eigenmodes reduces this expression to $\mathcal{E}=$ $\frac{1}{2} \sum_{s} \int_{0}^{2 \pi} N_{s \beta}\left|V_{s \beta}\right|^{2} \mathrm{~d}(\beta d)$. A similar calculation applies to the magnetic energy. Finally, using the inverse $\beta$ transform and the condition $N_{s \beta}=\Omega_{s \beta}$, we find that both expressions for the energy, $\mathcal{H}_{\mathrm{em}}$ and Poynting's, are equal:

$$
\frac{1}{2} \int_{\mathcal{V}_{\mathbb{Z}}}\left(\epsilon_{0}|\mathbf{E}|^{2}+\mu_{0}|\mathbf{H}|^{2}\right) \mathrm{d}^{3} \mathbf{r}=\frac{1}{2} \sum_{s}\left(V_{s} Q_{s} V_{s}^{t}+I_{s} Q_{s} I_{s}^{t}\right) .
$$

In this work, we constructed a Hamiltonian describing the electrons coupled with the electromagnetic fields propagating in a periodic structure. This coupled oscillator model may be viewed as a generalization of the telegraph delay line where each period consists of one inductor and capacitor, coupled with their nearest neighbors and electrons [14]. In contrast, in the present work all periods are coupled with each other. With this model, we can compute the time-dependent behavior of a helix traveling-wave tube, much faster than industrial PIC codes [3]. The reason is that the number of degrees of freedom to describe the propagating wave with sufficient accuracy in finite-difference time-domain or finite element techniques amounts to tens of thousands per pitch 11. In contrast, the beam is interacting with only one mode in a traveling wave tube, so that the number of degrees of fredom in the present theory is reduced to only one conjugate pair per pitch, the $\left(V_{n}, I_{n}\right)$ of the contemplated mode.

While we focused on divergence-free, propagating fields, the space charge field may be taken into account by adding the "space charge Hamiltonian" of two or more interacting electrons or by adding Darwin's first-order relativistic approximation [10, §12.6] (Green functions are needed to take into account the waveguide boundary condition). In this action-at-a-distance approach with two electrons interacting through their electrostatic field, the problem of infinite electromagnetic mass does not occur. This Hamiltonian is formally independent of time but depends explicitly on space through the $\mathbf{A}_{s n}(\mathbf{r})$. Indeed the boundary conditions - for example a corrugated metallic wall - enable the field to exchange momentum but not energy. Maxwell's equations and the Lorentz force correctly account for the recoil from the radiated field.

Self-acceleration can exist in the framework of a Hamiltonian description when it is the sum of two terms with opposite signs. In this case, the negative term of the system can indefinitely yield energy to the positive term while satisfying energy conservation. On the contrary, our Hamiltonian (24) is the sum of two unconditionally positive contributions which are minimum when the system is at rest, a situation precluding self-acceleration. As a consequence of the Hamiltonian, the source term in the equation of Maxwell-Ampère and the Lorentz force consistently express how the electron is coupled with the propagating field.

PB was supported by a CIFRE stipend from the French Ministère de l'Enseignement Supérieur et de la Recherche. NR enjoyed Thales' hospitality. A referee is warmly thanked for her/his recommendations. 
* corresponding author : frederic.andre@thalesgroup.com

[1] F. André and A. Aïssi, The equivalent matrices of a periodic structure, IEEE Trans. Electron Devices 57 (2010) 1687-1695.

[2] P. Bernardi, Utilisation et amélioration du modèle discret d'excitation d'un guide d'onde périodique pour la simulation pratique du tube à onde progressive en régime temporel (Ph.D. dissertation, univ. Provence, Marseille, 2011).

[3] P. Bernardi, F. André, J-F. David, A. Le Clair and F. Doveil, Efficient time domain simulation of a helix traveling-wave tube, IEEE Trans. Electron Devices 58 (2011) 1761-1767; Control of the reflections at the terminations of a slow wave structure in the non-stationary discrete theory of excitation of a periodic waveguide, IEEE Trans. Electron Devices 58 (2011) 4093-4097; Efficient 2.5-D non-stationary simulation of a helix TWT, Proc. IVEC, Bangalore, India 2011, pp. 307-308.

[4] P.A.M. Dirac, Classical theory of radiating electrons, Proc. R. Soc. London Ser. A 167 (1938) 148-169.

[5] I.M. Gel'fand, Expansion in characteristic functions of an equation with periodic coefficients (in Russian), Dokl. Akad. Nauk SSSR 73 (1950) 1117-1120.

[6] A.S. Gilmour Jr, Principles of traveling wave tubes (Artech House, Boston, 1994).

[7] E. Hairer, C. Lubich and G. Wanner, Geometric numerical integration: Structure-preserving algorithms for ordinary differential equations (Springer, New York, 2002).

[8] F. Hartemann, High-field electrodynamics (CRC Press, Boca Raton, 2002).

[9] W. Heitler, The quantum theory of radiation (Dover, New York, 1984).

[10] J.D. Jackson, Classical electrodynamics, 3rd ed. (Wiley, New York, 1999).

[11] M.V. Kartikeyan, E. Borie and M.K.A. Thumm, Gy- rotrons - High-power microwave and millimeter wave technology (Springer, Berlin, 2004).

[12] S.P. Kuznetsov, On one form of excitation equations of a periodic waveguide (in Russian), Sov. J. Comm. Technol. Electron. 25 (1980) 419-421.

[13] L.D. Landau and E.M. Lifshitz, Classical theory of fields, L.P. Pitaevskii ed., 4th English ed. (Elsevier, Amsterdam, 1975).

[14] J.R. Pierce, Interaction of moving charges with wave circuits, J. Appl. Phys. 26 (1955) 627-638.

[15] N.M. Ryskin, V.N. Titov, and A.V. Yakovlev, Nonstationary nonlinear discrete model of a coupled-cavity traveling wave tube amplifier, IEEE Trans. Electron Devices 56 (2009) 928-934.

[16] H. Spohn, Dynamics of charged particles and their radiation fields (Cambridge Univ. Press, Cambridge, UK, 2004).

[17] A. Taflove, Computational electrodynamics - The finitedifference time-domain method (Artech House, Boston, 1995).

[18] W. Thirring, Classical mathematical physics - Dynamical systems and field theories (Springer, New York 2003).

[19] A.I. Zayed, Zak transform, Encyclopedia of Mathematics, URL: http://www.encyclopediaofmath.org

[20] Some authors even suggested that the Lorentz force should be abandoned to recover consistency, e.g. M. Mansuripur, Trouble with the Lorentz law of force: Incompatibility with special relativity and momentum conservation, Phys. Rev. Lett. 108 (2012) 193901, with comments by D.A.T. Vanzella, ibid. 110 (2013) 089401, S.M. Barnett, ibid. 110 (2013) 089402, P.L. Saldanha, ibid. 110 (2013) 089403, M. Khorrami, ibid. 110 (2013) 089404, and author reply, ibid. 110 (2013) 089405. A solution starting from QED is considered by [21].

[21] B.A. van Tiggelen, S. Kawka, and G.L.J.A. Rikken, QED corrections to the electromagnetic Abraham force, Eur. Phys. J. D 66 (2012) 272. 